Research Paper

\title{
Expression of PD1/PDL1 in gastric cancer at different microsatellite status and its correlation with infiltrating immune cells in the tumor microenvironment
}

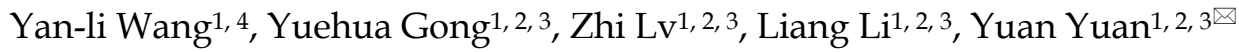 \\ 1. Tumor Etiology and Screening Department of Cancer Institute and General Surgery, the First Hospital of China Medical University, Shenyang 110001, \\ China. \\ 2. Key Laboratory of Cancer Etiology and Prevention in Liaoning Education Department, the First Hospital of China Medical University, Shenyang 110001, \\ China. \\ 3. Key Laboratory of GI Cancer Etiology and Prevention in Liaoning Province, the First Hospital of China Medical University, Shenyang 110001, China. \\ 4. Department of Medical Oncology, the First Affiliated Hospital of Jinzhou Medical University, Jinzhou 121001, China.
}

$\square$ Corresponding author: Dr. YuanYuan, Tumor Etiology and Screening Department of Cancer Institute and General Surgery, the First Hospital of China Medical University, No.155 NanjingBei Street, Heping District, Shenyang, Liaoning Province, P.R. China 110001. Telephone: +86-024-83282153; fax: +86-024-83282383. Email: yuanyuan@cmu.edu.cn

(C) The author(s). This is an open access article distributed under the terms of the Creative Commons Attribution License (https://creativecommons.org/licenses/by/4.0/). See http://ivyspring.com/terms for full terms and conditions.

Received: 2019.09.21; Accepted: 2020.12.29; Published: 2021.01.18

\begin{abstract}
Objective: The microsatellite status and tumor immune microenvironment have a remarkable influence on tumor immunotherapy. This study was performed to investigate programmed cell death protein 1/programmed death ligand 1 (PDI/PDLI) expression and their correlations with CD8+ T cell/CD68+ macrophage (CD68+ M) densities in gastric cancer (GC) at different microsatellite statuses.

Methods: The expression of MLH1, PMS2, MSH2, and MSH6 was detected via immunohistochemistry (IHC) to determine the microsatellite status in $215 \mathrm{GC}$ samples obtained from surgical resections. Furthermore, the expression of PDI, PDLI, CD8, and CD68 was detected in the samples via IHC, and the differences and correlations in GC at different microsatellite statuses were then analyzed. PDL1 expression in tumor cells was labeled as PDLI[T], while expression of PDI and PDLI in tumor-infiltrating immune cells was labeled as PDI and PDLI, respectively. Kaplan-Meier analysis was used to evaluate the significance of PDI/PDL1 expression in determining overall survival. Multivariate Cox regression analysis was performed using SPSS software. P-values were determined using the log-rank test.

Results: Our results indicated that PDI, PDLI [T], and PDL1 positivity rates were $59 \%, 35 \%$, and $57 \%$ in 46 microsatellite unstable (MSI) GCs and $45 \%, 22 \%$, and $40 \%$ in 169 microsatellite stable (MSS) GCs, respectively. Compared with MSS GC, PDI, PDL1[T], and PDL1 expression was higher in MSI GC (P = $0.109,0.090$, and 0.044 , respectively). Additionally, CD8+ T cell and CD68+ M densities were higher in MSI GC than in MSS GC $(P=0.537$ and $<0.001$, respectively). Additionally, CD8+ $T$ cell/CD68+ $M$ densities were evaluated according to tumor center and invasion front. We found that PDI expression was significantly correlated with CD8+ T cell density at the invasion front of the MSI GC $(P=0.031)$, whereas PDLI expression was significantly correlated to high CD68+ M density in the tumor center and invasion front of MSS GC $(P=0.001$ and 0.014 , respectively). Survival analysis showed that patients with PDI-positive and PDLI[T]/PDLI-negative GC had better prognosis $(P=0.012,0.005$, and 0.022 , respectively). Multivariate Cox survival analysis showed that PDLI[T] was an independent prognostic factor for GC.

Conclusion: The results suggested that PDI/PDL1 expression and immune response varied at different microsatellite statuses in GC. PD1/PDL1 expression was correlated with CD8+ $T$ cell/CD68+ $M$ densities in GC at different microsatellite statuses, especially at the invasion front. The patients exhibiting high PDI/PDLI expression or high CD8+ $T$ cell/CD68+ $M$ densities MSI GC might be potential beneficiaries of PDI/PDLI immunotherapy.
\end{abstract}

Key words: gastric cancer, tumor immune microenvironment, PD1, PDL1, CD8, CD68 


\section{Introduction}

Gastric cancer (GC) is the fifth most frequently diagnosed malignancy and the third leading cause of cancer-related death worldwide [1]. Four molecular classifications of GC were proposed according to the comprehensive molecular characterization by TCGA, including Epstein-Barr virus-positive GC, microsatellite unstable GC (MSI), genomically stable GC, and chromosomal instability [2]. As one of the four molecular subtypes, MSI GC accounts for $21.7 \%$ of all GCs and is caused due to the misfunction of the DNA mismatch repair (MMR) system. Compared with other molecular types, MSI GC has special characteristics, such as formation mechanisms, molecular compositions, immune microenvironments, and clinicopathological phenotypes. Knowledge of the unique biological characteristics of MSI GC is critical for its precise diagnosis and treatment.

Mounting evidence has shown that the tumor microenvironment plays an essential role in the progression of many solid tumors; tumor microenvironment is mainly orchestrated by tumorinfiltrating immune cells, including neutrophils, dendritic cells, macrophages, eosinophils, mast cells, and lymphocytes. The tumor microenvironment is indispensable for tumor progression, fostering proliferation, survival, and migration [3]. Tumor-infiltrating lymphocytes (TILs, mainly CD8+ T lymphocytes) constitute the most essential effector cells of antitumor immunity [4]. Tumor-associated macrophages (TAMs, which can be labeled by CD68 as CD68+ M) are also a significant component of tumor-infiltrating immune cells [5]. Tumorinfiltrating immune cells and tumor cells interact with each other to maintain the balance of the tumor microenvironment. Therefore, knowledge of the heterogeneity of the tumor microenvironment, especially the immunocyte phenotypes related to therapeutic targets, can provide clues for new treatments for GC.

As one of the most promising immunotherapy approaches, programmed cell death protein 1 (PD1) and its ligand, programmed death ligand 1 (PDL1), have been used in the treatment of a variety of tumors in recent years [6-8]. PD1, a member of the CD28 superfamily, is an important immunosuppressive molecule, mainly expressed in $\mathrm{T}, \mathrm{NK}$, and $\mathrm{B}$ cells surrounding tumor tissues [9]. PDL1 is a $40 \mathrm{kDa}$ type I transmembrane protein that is extensively expressed in immune, tumor, epithelial, and endothelial cells. In the tumor microenvironment, PDL1 is expressed in tumor cells interacting with PD1 expressed in T cells and inhibits the function of $\mathrm{T}$ cells. PD1/PDL1 immunotherapy can prevent the recognition between PD1 and PDL1, thus restoring the normal function of
$\mathrm{T}$ cells $[10,11]$. Based on previous publications, the response rate to PD1/PDL1 immunotherapy appears to be associated with PDL1 expression levels $[12,13]$. Currently, the detection of PD1/PDL1 protein expression via immunohistochemistry (IHC) is routinely performed to select patients who will benefit from PD1/PDL1 immunotherapy [14]. It has been found that $36 \%$ to $100 \%$ of patients with PDL1-positive tumor cells, detected via IHC, respond to PD1/PDL1 therapy; however, $0 \%$ to $17 \%$ of PDL1-negative patients also respond [15], suggesting that additional factors may be involved in predicting the response. Patients who could benefit the most from PD1/PDL1 immunotherapy required laminated anchoring. Therefore, additional biomarkers need to be further investigated, especially in the tumor immune microenvironment.

So far, the relationship among MSI characteristics of tumors, heterogeneity of the tumor microenvironment, and PD1/PDL1 immunotherapy has attracted increasing attention in clinical studies. A basic consensus has been reached to identify MSI status and PD1/PDL1 expression to select patients who would benefit from PD1/PDL1 immunotherapy $[8,14,16,17]$. However, PD1/PDL1 expression and its correlation with immune cell phenotypes in MSI GC remains unclear, as does the cells targeted by PD1/PDL1 immunotherapy. This study evaluated PD1/PDL1 expression in the microenvironment of MSI GC and analyzed its correlation with immune cell phenotypes to identify the cell types related to PD1/PDL1 expression in the microenvironment of MSI GC to better understand the interactions between tumor and immune cells and help select suitable target populations for future PD1/PDL1 immunotherapy in GC with different microsatellite statuses.

\section{Materials and Methods}

\section{Patients and clinical data collection}

The samples and data of 215 patients who underwent surgical resection of primary GC at the first hospital of China Medical University and Jinzhou Medical University of Liaoning province between January 2012 and December 2018 were collected retrospectively. None of the patients had undergone neoadjuvant therapy. The histological diagnosis was confirmed by two pathologists independently, according to the World Health Organization criteria. Pathological Pathological Tumor Node Metastasis (TNM) staging was classified based on the Union for International Cancer Control (UICC; 8th edition, 2017). The study was approved by the Ethics Committee of the First Affiliated Hospital of China 
Medical University and Jinzhou Medical University. All participants enrolled in the study signed written informed consent forms.

\section{Immunohistochemical staining and evaluation}

\subsection{Immunohistochemical staining}

The total number of cases in this study was 215 . For each case, 9 consecutive sections were made for HE (Hematoxylin - Eosin) staining and immunohistochemical staining of 8 indexes including MLH1, MSH2, PMS2, MSH6, CD8, CD68, PDL1 and PD1. Because a few cases tissue is too little to the last 2-3 tissue section to complete the immunohistochemical evaluation. So some individual index staining is less than the total number of all cases. MLH1, MSH2, MSH6 and PMS2 evaluations were completed in all 215 cases, and 205 cases were evaluable for PD1, 202 cases for PDL1 [T]/PDL1, 205 cases for CD8, and 202 cases for CD68.

The primary antibodies used in this study (including anti-MLH1, -PMS2, -MSH2, -MSH6, -CD8, and -CD68 antibodies), DAB chromogenic solution, and UltraSensitive TMSP detection kit were purchased from Maixin (Maixin Inc., Fujian, China). Anti-PDL1 antibody [28-8] (ab205921), anti-PD1 antibody [EPR4877(2)] (ab137132), and Universal HIER antigen retrieval reagent (10X) (ab208572) were purchased from Abcam (Cambridge, UK).

IHC was performed on $4 \mu \mathrm{m}$-thick tissue sections mainly as previously described [18] at room temperature $\left(18-30{ }^{\circ} \mathrm{C}\right)$ according to manufacturer's instructions. The primary antibodies (anti-MLH1, -PMS2, -MSH2, -MSH6, -CD8, and -CD68 antibodies) were ready for use. Anti-PD1 and anti-PDL1 antibodies were diluted at 1:260 and 1:600, respectively. Phosphate buffer solution (PBS) was used as the negative control, and positive staining of normal gastric interstitial immune cells was the internal control (MLH1, PMS2, MSH2, and MSH6).

\subsection{Immunohistochemical evaluation}

Each section was first observed under a low-power microscope for the entire area. Positive staining areas were selected and observed under a high-power microscope. The evaluation was independently conducted by two senior pathologists.

\subsubsection{Microsatellite status assessment}

MLH1, PMS2, MSH2, and MSH6 were all located in the GC cell nucleus. The tumor cell nuclei in brown yellow or brown granules were determined to be positive staining. Absence was determined when the tumor cell nucleus failed to stain. Microsatellite stability was determined when four proteins were positive, whereas MSI was determined when one or more proteins were absent. The microsatellite status was determined consistently by evaluating the expression of MLH1, PMS2, MSH2, and MSH6 in GC and adjacent peritumoral tissues simultaneously, although there were significant differences in the expression levels between them.

\subsubsection{PDI and PDL1 expression evaluation}

PD1 and PDL1 were located in the membrane and/or cytoplasm. PDL1 expression in tumor cells was labeled as PDL1[T], and expression of PD1 and PDL1 in tumor-infiltrating immune cells was labeled as PD1 and PDL1, respectively. We randomly selected five high-power fields (HPF, $\times 400,0.025 \mathrm{~mm}^{2}$ ) from each sample, and manually counted 100 tumor or immune cells in each HPF and then calculated the percentage of positively stained cells. The mean value was adopted as the positive expression rate of PD1, PDL1[T], and PDL1. Positive staining was defined as more than $1 \%$ stained cells, regardless of cytoplasmic or membrane staining $[14,19]$.

\subsubsection{CD8 and CD68 expression evaluation}

We randomly selected five HPFs $(\times 400,0.025$ $\mathrm{mm}^{2}$ ) in the tumor center and invasive front, respectively, for each case, then manually counted and calculated the average number of stained CD8+ T cells and CD68+ $\mathrm{M}$ according to the median number of stained cells (CD8, 72.3/HPF; CD68, 43.2/HPF). Patients were divided into two groups: high and low density.

\section{Statistical analysis}

All statistical analyses were performed using SPSS software, Chicago, IL, USA (version 23.0). Intergroup comparison (associations between PD1/PDL1-positive expression, CD8/CD68-positive cell density, and MSI/MSS) was conducted using the X2 test or Fisher's exact test. Kaplan-Meier analysis was used to evaluate the significance of different factors in determining overall survival. Multivariate Cox regression analysis was performed using SPSS software. P-values were determined using the log-rank test. $\mathrm{P}<0.05$ was considered statistically significant.

\section{Results}

\section{Expression of MLH1, PMS2, MSH2, and MSH6 in GC and microsatellite status judgment}

Based on the expression of MLH1, PMS2, MSH2, and MSH6, 215 samples were divided into two groups: 46 MSI (21.4\%) and 169 microsatellite stable (MSS) (78.6\%) (Table 1 and Figure 1). 
Table 1 Expression of MLH1, PMS2, MSH2 and MSH6 and microsatellite status in GC.

\begin{tabular}{lllllll}
\hline MLH1 & PMS2 & MSH2 & MSH6 & Samples (\%) & MSI status & sum \\
\hline+ & + & + & + & $169(78.6)$ & MSS & 169 \\
- & + & + & + & $6(2.8)$ & & \\
+ & - & + & + & $22(10.2)$ & & \\
+ & + & - & + & $1(0.5)$ & MSI & 46 \\
+ & + & + & - & $7(3.3)$ & & \\
- & - & + & + & $10(4.6)$ & & \\
\hline
\end{tabular}

GC, gastric cancer; MSI, microsatellite instability; MSS, microsatellite stability. MLH1, PMS2, MSH2 and MSH6 immunohistochemical evaluations were completed in all 215 cases.

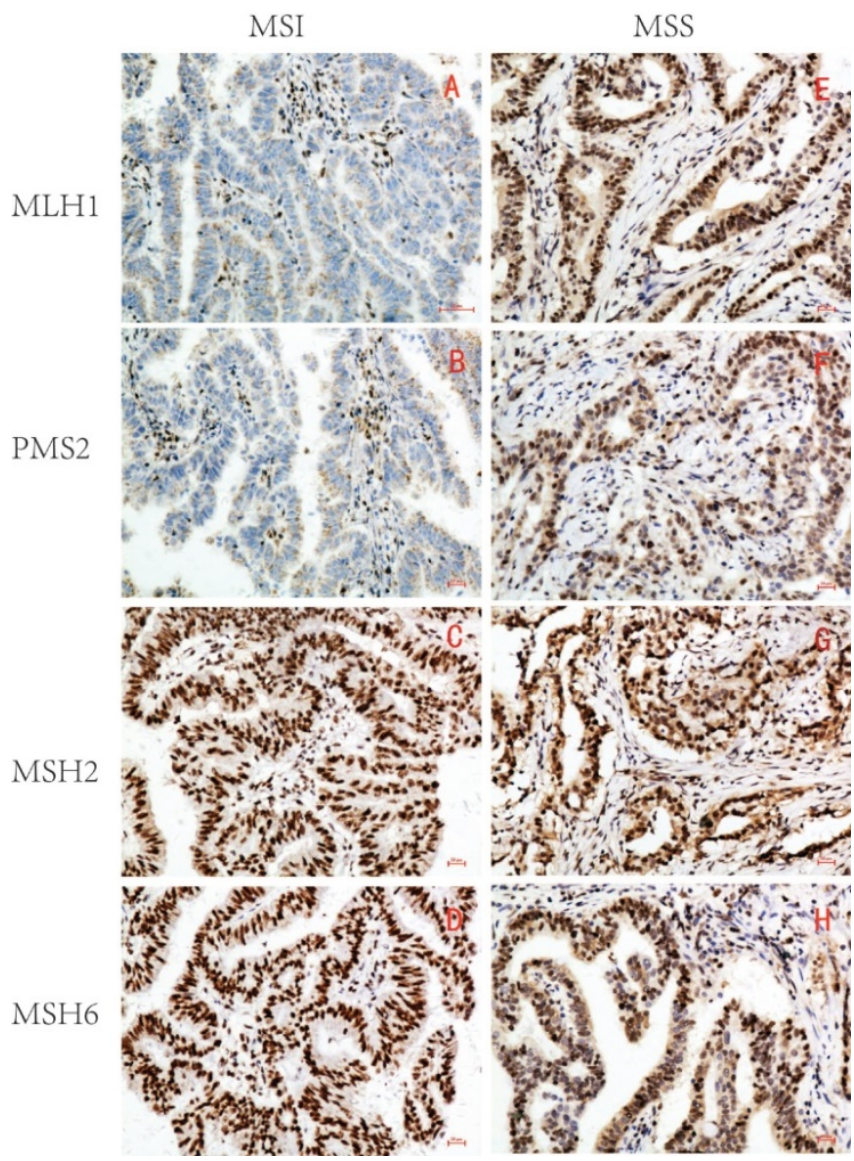

Figure 1 Representative immunohistochemical images of MSI/MSS GC $(\times 200)$. MLH1, PMS2, MSH2 and MSH6 were all localized in GC cell nucleus. The brown yellow was considered positive, while the absence of staining was considered negative. MSI GC: $M L H 1$ and PMS2 were negative(A,B), MSH2 and MSH6 were positive(C,D); MSS GC: MLH1, PMS2, MSH2 and MSH6 were all positive(E-H).

\section{PD1/PDLI expression in MSI and MSS GC}

PD1, PDL1[T], and PDL1 expression rates were $59 \%, 35 \%$, and $57 \%$ in 46 MSI GC and $45 \%, 22 \%$, and $40 \%$ in 169 MSS GC, respectively. Statistical analysis showed that the PDL1 expression rate in MSI GC was significantly higher than in MSS GC $(P=0.044)$, while the PD1/PDL1[T] expression rate in MSI GC was higher than in MSS GC but without statistical significance $(\mathrm{P}=0.109$ and 0.090$)$ (Table 2 and Figure 2).
Table 2 PD1/PDL1 expression and CD8+T cells/CD68+M density in MSI and MSS GC

\begin{tabular}{lllll}
\hline & MSI (\%) & MSS (\%) & Sum & P \\
\hline PD1 & & & & 0.109 \\
Negative & $19(41)$ & $87(55)$ & 106 & \\
Positive & $27(59)$ & $72(45)$ & 99 & \\
Sum & $46(100)$ & $159(100)$ & 205 & \\
PDL1 [T] & & & & 0.090 \\
Negative & $30(65)$ & $121(78)$ & 151 & \\
Positive & $16(35)$ & $35(22)$ & 51 & \\
Sum & $46(100)$ & $156(100)$ & 202 & \\
PDL1 & & & & 0.044 \\
Negative & $20(43)$ & $94(60)$ & 114 & \\
Positive & $26(57)$ & $62(40)$ & 88 & \\
Sum & $46(100)$ & $156(100)$ & 202 & \\
CD8 & & & & 0.537 \\
Low & $21(47)$ & $83(52)$ & 104 & \\
High & $24(53)$ & $77(48)$ & 101 & \\
Sum & $45(100)$ & $160(100)$ & 205 & \\
CD68 & & & & $<0.001$ \\
Low & $7(16)$ & $95(60)$ & 102 & \\
High & $37(84)$ & $63(40)$ & 100 & \\
Sum & $44(100)$ & $158(100)$ & 202 & \\
\hline
\end{tabular}

GC: gastric cancer; MSI: microsatellite unstable; MSS: microsatellite stable; CD68+ M: CD68+ macrophage; PD1: programmed cell death protein 1; PDL1, programmed death ligand 1; In all 215 cases of gastric cancer, 205 cases immunohistochemical sections were evaluable for PD1, 202 cases for PDL1 [T]/PDL1, 205 cases for CD8, and 202 cases for CD68

\section{3. $C D 8+T$ cell/CD68+ $M$ densities in $M S I$ and MSS GC}

According to the median number of stained cells (CD8, 72.3/HPF; CD68, 43.2/HPF), patients were divided into two groups: high and low density. CD8+ $\mathrm{T}$ cell density in MSI GC was higher than in MSS GC but without statistical significance $(\mathrm{P}=0.537)$. $\mathrm{CD68+}$ $M$ density in MSI GC was significantly higher than that in MSS GC $(\mathrm{P}<0.001)$ (Table 2 and Figure 2).

\section{Correlations between PD1/PDLI expression and CD8+ $T$ cell/CD68+ $M$ densities in MSI and MSS GC}

PD1 expression rate in the high CD8+ $\mathrm{T}$ cell density group was greater than that in the low-density group in MSI GC but without statistical significance $(P=0.058)$. PDL1 expression rate in the high CD68+ M density group was greater than that in the low-density group in MSS GC $(P=0.013)$. However, PDL1[T] expression was not correlated with CD8+ T cell/CD68+ M densities in MSI or MSS GC (Table 3).

\section{Correlations between PD1/PDL1 expression and $C D 8+T$ cell/CD68+ $M$ densities at different locations in MSI and MSS GC}

PD1 expression was significantly correlated with CD8+ T cell density at the invasion front of MSI GC (P $=0.031)$. PDL1 expression was significantly correlated with high $\mathrm{CD} 68+\mathrm{M}$ density in the tumor center and invasion front of MSS GC $(\mathrm{P}=0.001$ and 0.014, 
respectively). However, PDL1[T] expression was not correlated with CD8+ $\mathrm{T}$ cell/CD68+ $\mathrm{M}$ densities in the tumor center and invasion front of MSI or MSS GC (Table 4, 5 and Figure 3).

\section{Correlations between PD1/PDLI expression or $C D 8+T$ cell/CD68+ $M$ densities and prognosis in GC}

Survival analysis showed that patients with MSI who were PD1 positive, PDL1[T] negative, and PDL1 negative had a better $\mathrm{GC}$ prognosis $(\mathrm{P}=0.006,0.012$, 0.005 , and 0.022 , respectively). There were no correlations between CD8+ $\mathrm{T}$ cell/CD68+ $\mathrm{M}$ densities and prognosis in GC $(\mathrm{P}=0.870$ and 0.985, respectively). Multivariate survival analysis showed that microsatellite status, CD8+ $\mathrm{T}$ cell density, PDL1[T], TNM stage, infiltration depth, and vascular thrombi were all independent prognostic factors for GC.

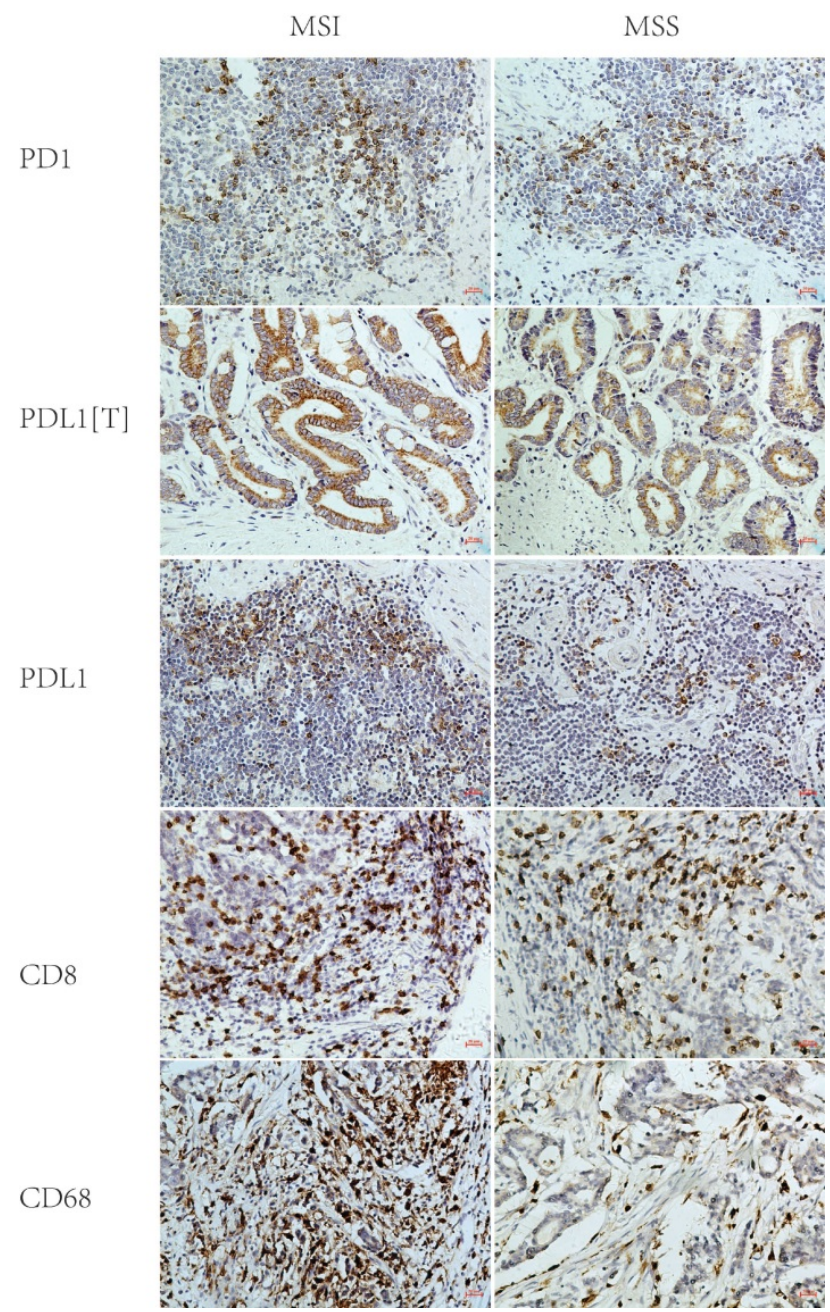

Figure 2 Representative immunohistochemical images of PD1, PDL1 [T], PDL1, CD8 and CD68 in MSI and MSS GC (×200). PD1, PDL1 [T], PDL1, CD8 and CD68 were all localized in the membrane and/or cytoplasm. PDI, CD8 and CD68 was all stained in tumor infiltrating immune cells, while PDL1 was stained in tumor cells (labeled as PDL1[T]) and tumor infiltrating immune cells (labeled as PDL1). PDI, PDL1[T] and PDL1 expression in MSI GC was greater than those in MSS GC $(P=0.109,0.090$ and 0.044 , respectively). CD8+T cells/CD68+M density in MSI GC was higher than those in MSS GC ( $\mathrm{P}=0.537$ and $<0.001$, respectively).

\section{Discussion}

In this study, we investigated PD1/PDL1 expression and its correlations with infiltrating immune cells in GC with different microsatellite statuses. It was found that both PD1/PDL1 expression and CD8+ $\mathrm{T}$ cell/CD68+ $\mathrm{M}$ densities were higher in MSI GC than in MSS GC. Furthermore, PD1/PDL1 expression was significantly correlated with high CD8+ $\mathrm{T}$ cell/CD68+ $\mathrm{M}$ densities, especially at the invasion front. These results may provide new information regarding the accurate stratification of target populations for PD1/PDL1 immunotherapy in GC.

A microsatellite is a simple, repetitive sequence evenly distributed in eukaryotic genomes under normal conditions. MSI refers to any change in the length of microsatellites resulting from insertion or deletion of repetitive units in a tumor and usually arises from germline mutations in the components of MMR genes (mainly MLH1, PMS2, MSH2, and MSH6) [20]. Based on the expression of MLH1, PMS2, MSH2, and MSH6, 46 MSI GCs were detected in 215 GCs in this study; the proportion of MSI GC $(21.40 \%)$ was similar to that in TCGA classification [2]. We found that PD1/PDL1 expression in MSI GC was higher than that in MSS GC, suggesting that the immune response varied from microsatellite status in GC. Previous studies have reported that PD1/PDL1 immunotherapy shows little activity in unselected cancer [13, 21]; however, significant clinical responses have been observed in patients characterized by MMR deficiency $[16,17]$. After radical surgery, patients with GC should be stratified and selected for postoperative adjuvant therapy. PD1 immunotherapy is a treatment option for chemotherapy-refractory GC, especially for PDL1-positive or MSI-High cancers [22, 23]. PDL1 expression in tumor cells appears to be more common in MSI than in MSS GC [24, 25]. Therefore, selecting beneficiaries of PD1/PDL1 immunotherapy according to microsatellite status in GC is a reasonable strategy.

The tumor microenvironment is primarily composed of TILs, especially CD8+ T cells and CD68+ TAMs. It has previously been reported that tumors with different microsatellite statuses may exhibit different immune microenvironments. MSI is associated with higher density TILs, possibly in response to the new environment created by neoantigens [26]. Shin et al. found that the density of each subgroup of TILs varied from the microsatellite status of GC, and CD8+ T cell density was highly increased in MSI-H GC [27, 28]. MSI-H GC was significantly correlated with CD8 $+\mathrm{T}$ cells [29]. Furthermore, MSI tumors had more CD8+ T cell/CD68+ $\mathrm{M}$ in the epithelium, in contrast to MSS tumors [30]. Intraepithelial CD68+ $\mathrm{M}$ density was 
significantly correlated with MSI [31]. Additionally,

immune microenvironment was significantly we found that CD8+ T cell/CD68+ M densities were different between MSI and MSS GC.

higher in MSI GC than in MSS GC, suggesting that the

Table 3 The correlations between PDI/PDL1 expression and CD8+T cells/CD68+M density in MSI and MSS GC

\begin{tabular}{|c|c|c|c|c|c|c|c|c|c|c|c|c|c|c|c|c|}
\hline & MSI & & & & & & & & MSS & & & & & & & \\
\hline & CD8 & & & & CD68 & & & & CD8 & & & & CD68 & & & \\
\hline & $\begin{array}{l}\text { Low } \\
(\%)\end{array}$ & $\begin{array}{l}\text { High } \\
(\%)\end{array}$ & Sum & $\mathrm{P}$ & $\begin{array}{l}\text { Low } \\
(\%)\end{array}$ & $\begin{array}{l}\text { High } \\
(\%)\end{array}$ & Sum & $\mathrm{P}$ & $\begin{array}{l}\text { Low } \\
(\%)\end{array}$ & $\begin{array}{l}\text { High } \\
(\%)\end{array}$ & Sum & $\mathrm{P}$ & $\begin{array}{l}\text { Low } \\
(\%)\end{array}$ & $\begin{array}{l}\text { High } \\
(\%)\end{array}$ & Sum & $\mathrm{P}$ \\
\hline PD1 & & & & 0.058 & & & & 0.443 & & & & 0.086 & & & & 0.405 \\
\hline Negative & $12(57)$ & $7(29)$ & 19 & & $4(57)$ & $15(41)$ & 19 & & $50(61)$ & $36(47)$ & 86 & & $54(57)$ & $30(50)$ & 84 & \\
\hline Positive & $9(43)$ & $17(71)$ & 26 & & $3(43)$ & $22(59)$ & 25 & & $32(39)$ & $40(53)$ & 72 & & $41(43)$ & $30(50)$ & 71 & \\
\hline Sum & $21(100)$ & $24(100)$ & 45 & & $7(100)$ & $37(100)$ & 44 & & $82(100)$ & $76(100)$ & 158 & & $95(100)$ & $60(100)$ & 155 & \\
\hline PDL1[T] & & & & 1.000 & & & & 0.401 & & & & 0.620 & & & & 0.472 \\
\hline Negative & $14(67)$ & $16(67)$ & 30 & & $6(86)$ & $24(65)$ & 30 & & $64(79)$ & $56(76)$ & 120 & & $74(80)$ & $44(75)$ & 118 & \\
\hline Positive & $7(33)$ & $8(33)$ & 15 & & $1(14)$ & $13(35)$ & 14 & & $17(21)$ & $18(24)$ & 35 & & $19(20)$ & $15(25)$ & 34 & \\
\hline Sum & $21(100)$ & $24(100)$ & 45 & & $7(100)$ & $37(100)$ & 44 & & $81(100)$ & $74(100)$ & 155 & & $93(100)$ & $59(100)$ & 152 & \\
\hline PDL1 & & & & 0.688 & & & & 0.217 & & & & 0.844 & & & & 0.013 \\
\hline Negative & $10(48)$ & $10(42)$ & 20 & & $5(71)$ & $15(41)$ & 20 & & $48(59)$ & $45(61)$ & 93 & & $63(68)$ & $28(48)$ & 91 & \\
\hline Positive & $11(52)$ & $14(58)$ & 25 & & $2(29)$ & $22(59)$ & 24 & & $33(41)$ & 29(39) & 62 & & $30(32)$ & $31(52)$ & 61 & \\
\hline Sum & $21(100)$ & $24(100)$ & 45 & & $7(100)$ & $37(100)$ & 44 & & $81(100)$ & $74(100)$ & 155 & & $93(100)$ & $59(100)$ & 152 & \\
\hline
\end{tabular}

GC: gastric cancer; MSI: microsatellite unstable; MSS: microsatellite stable; CD68+ M: CD68+ macrophage; PD1: programmed cell death protein 1; PDL1, programmed death ligand 1; In all 215 cases of gastric cancer, 203 cases immunohistochemical sections were evaluable for PD1 and CD8 simultaneously, 200 cases for PDL1 [T]/PDL1 and CD8 simultaneously, 199 cases for PD1 and CD68 simultaneously, and 196 cases for PDL1 [T]/PDL1 and CD68 simultaneously.

Table 4 The correlations between PDI/PDL1 expression and CD8+T cells density at different locations in MSI and MSS GC

\begin{tabular}{|c|c|c|c|c|c|c|c|c|c|c|c|c|c|c|c|c|}
\hline & \multicolumn{8}{|c|}{ MSI-CD8 } & \multicolumn{8}{|c|}{ MSS-CD8 } \\
\hline & \multicolumn{4}{|c|}{ Tumor center (\%) } & \multicolumn{4}{|c|}{ Invasive front (\%) } & \multicolumn{4}{|c|}{ Tumor center (\%) } & \multicolumn{4}{|c|}{ Invasive front (\%) } \\
\hline & $\begin{array}{l}\text { Low } \\
(\%)\end{array}$ & $\begin{array}{l}\text { High } \\
(\%)\end{array}$ & Sum & $P$ & $\begin{array}{l}\text { Low } \\
(\%)\end{array}$ & $\begin{array}{l}\text { High } \\
(\%)\end{array}$ & Sum & $P$ & $\begin{array}{l}\text { Low } \\
(\%)\end{array}$ & $\begin{array}{l}\text { High } \\
(\%)\end{array}$ & Sum & $P$ & $\begin{array}{l}\text { Low } \\
(\%)\end{array}$ & $\begin{array}{l}\text { High } \\
(\%)\end{array}$ & Sum & $\mathrm{P}$ \\
\hline PD1 & & & & 0.550 & & & & 0.031 & & & & 0.381 & & & & 0.163 \\
\hline Negative & $9(47)$ & $10(39)$ & 19 & & $12(60)$ & $7(28)$ & 19 & & $49(58)$ & $37(51)$ & 86 & & $49(60)$ & $37(49)$ & 86 & \\
\hline Positive & $10(53)$ & $16(61)$ & 26 & & $8(40)$ & $18(72)$ & 26 & & $36(42)$ & $36(49)$ & 72 & & $33(40)$ & $39(51)$ & 72 & \\
\hline Sum & 19(100) & $26(100)$ & 45 & & $20(100)$ & $25(100)$ & 45 & & $85(100)$ & 73(100) & 158 & & $82(100)$ & $76(100)$ & 158 & \\
\hline PDL1[T] & & & & 0.831 & & & & 0.832 & & & & 0.691 & & & & 0.427 \\
\hline Negative & $13(68)$ & $17(65)$ & 30 & & $13(65)$ & $17(68)$ & 30 & & $64(76)$ & $56(79)$ & 120 & & $64(80)$ & $56(75)$ & 120 & \\
\hline Positive & $6(32)$ & $9(35)$ & 15 & & $7(35)$ & $8(32)$ & 15 & & $20(24)$ & $15(21)$ & 35 & & $16(20)$ & $19(25)$ & 35 & \\
\hline Sum & 19(100) & $26(100)$ & 45 & & $20(100)$ & $25(100)$ & 45 & & $84(100)$ & 71(100) & 155 & & $80(100)$ & $75(100)$ & 155 & \\
\hline PDL1 & & & & 0.345 & & & & 0.947 & & & & 0.148 & & & & 0.743 \\
\hline Negative & $10(53)$ & 10(39) & 20 & & $9(45)$ & $11(44)$ & 20 & & $46(55)$ & $47(66)$ & 93 & & $49(61)$ & $44(59)$ & 93 & \\
\hline Positive & $9(47)$ & $16(61)$ & 25 & & 11(55) & $14(56)$ & 25 & & $38(45)$ & $24(34)$ & 62 & & $31(39)$ & $31(41)$ & 62 & \\
\hline Sum & 19(100) & $26(100)$ & 45 & & $20(100)$ & $25(100)$ & 45 & & $84(100)$ & 71(100) & 155 & & $80(100)$ & $75(100)$ & 155 & \\
\hline
\end{tabular}

GC: gastric cancer; MSI: microsatellite unstable; MSS: microsatellite stable; CD68+ M: CD68+ macrophage; PD1: programmed cell death protein 1; PDL1, programmed death ligand 1; In all 215 cases of gastric cancer, 203 cases immunohistochemical sections were evaluable for PD1 and CD8 simultaneously in tumor center and invasive front, 200 cases for PDL1 [T]/PDL1 and CD8 simultaneously in tumor center and invasive front.

Table 5 The correlations between PDI/PDL1 expression and CD68+M cells density at different locations in MSI and MSS GC

\begin{tabular}{|c|c|c|c|c|c|c|c|c|c|c|c|c|c|c|c|c|}
\hline & \multicolumn{8}{|c|}{ MSI-CD68 } & \multicolumn{8}{|c|}{ MSS-CD68 } \\
\hline & \multicolumn{4}{|c|}{ Tumor center (\%) } & \multicolumn{4}{|c|}{ Invasive front (\%) } & \multicolumn{4}{|c|}{ Tumor center (\%) } & \multicolumn{4}{|c|}{ Invasive front (\%) } \\
\hline & $\begin{array}{l}\text { Low } \\
(\%)\end{array}$ & $\begin{array}{l}\text { High } \\
(\%)\end{array}$ & Sum & $\mathrm{P}$ & $\begin{array}{l}\text { Low } \\
(\%)\end{array}$ & $\begin{array}{l}\text { High } \\
(\%)\end{array}$ & Sum & $\mathrm{P}$ & $\begin{array}{l}\text { Low } \\
(\%)\end{array}$ & $\begin{array}{l}\text { High } \\
(\%)\end{array}$ & Sum & $\mathrm{P}$ & $\begin{array}{l}\text { Low } \\
(\%)\end{array}$ & $\begin{array}{l}\text { High } \\
(\%)\end{array}$ & Sum & $\mathrm{P}$ \\
\hline PD1 & & & & 1.000 & & & & 0.051 & & & & 0.708 & & & & 0.292 \\
\hline Negative & $4(40)$ & $15(44)$ & 19 & & $7(70)$ & $12(35)$ & 19 & & $51(55)$ & $33(52)$ & 84 & & $52(58)$ & $32(49)$ & 84 & \\
\hline Positive & $6(60)$ & $19(56)$ & 25 & & $3(30)$ & $22(65)$ & 25 & & $41(45)$ & $30(48)$ & 71 & & $38(42)$ & $33(51)$ & 71 & \\
\hline Sum & $10(100)$ & $34(100)$ & 44 & & $10(100)$ & $34(100)$ & 44 & & $92(100)$ & $63(100)$ & 155 & & $90(100)$ & $65(100)$ & 155 & \\
\hline PDL1[T] & & & & 0.361 & & & & 0.361 & & & & 0.102 & & & & 0.290 \\
\hline Negative & $8(80)$ & $22(65)$ & 30 & & $8(80)$ & $22(65)$ & 30 & & $74(82)$ & $44(71)$ & 118 & & $71(81)$ & $47(73)$ & 118 & \\
\hline Positive & $2(20)$ & $12(35)$ & 14 & & $2(20)$ & $12(35)$ & 14 & & $16(18)$ & $18(29)$ & 34 & & 17(19) & $17(27)$ & 34 & \\
\hline Sum & $10(100)$ & $34(100)$ & 44 & & $10(100)$ & $34(100)$ & 44 & & $90(100)$ & $62(100)$ & 152 & & $88(100)$ & $64(100)$ & 152 & \\
\hline PDL1 & & & & 0.743 & & & & 0.147 & & & & 0.001 & & & & 0.014 \\
\hline Negative & $5(50)$ & $15(44)$ & 20 & & $7(70)$ & $13(38)$ & 20 & & $64(71)$ & $27(44)$ & 91 & & $60(68)$ & $31(48)$ & 91 & \\
\hline Positive & $5(50)$ & $19(56)$ & 24 & & $3(30)$ & $21(62)$ & 24 & & $26(29)$ & $35(56)$ & 61 & & $28(32)$ & $33(52)$ & 61 & \\
\hline Sum & $10(100)$ & $34(100)$ & 44 & & $10(100)$ & $34(100)$ & 44 & & $90(100)$ & $62(100)$ & 152 & & $88(100)$ & $64(100)$ & 152 & \\
\hline
\end{tabular}

GC: gastric cancer; MSI: microsatellite unstable; MSS: microsatellite stable; CD68+ M: CD68+ macrophage; PD1: programmed cell death protein 1; PDL1, programmed death ligand 1; In all 215 cases of gastric cancer, 199 cases immunohistochemical sections were evaluable for PD1 and CD68 simultaneously in tumor center and invasive front, 196 cases for PDL1 [T]/PDL1 and CD68 simultaneously in tumor center and invasive front. 

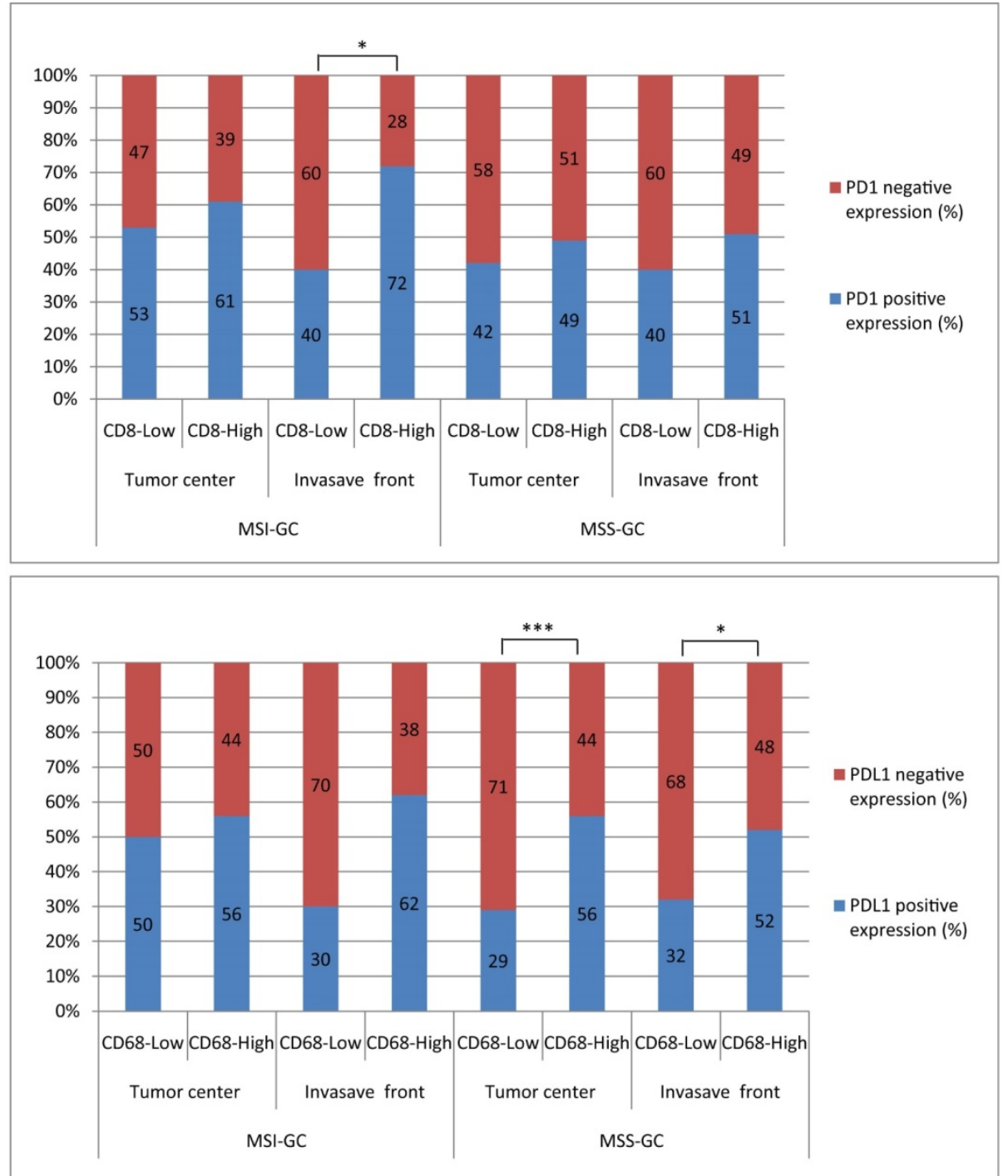

Figure 3 The correlations between PDI/PDL1 expression and CD8+T cells/CD68+M density at different locations in MSI and MSS GC. PDI expression was significantly related to $C D 8+T$ cells high density at invasion front of MSI GC $(P=0.031)$. PDL1 expression was significantly related to $C D 68+M$ high density in tumor center and invasion front of $M S S$ $\mathrm{GC}(\mathrm{P}=0.001$ and 0.014 , respectively).

Table 6 Multivariate Cox regression analysis of the association of various clinicopathological features with overall survival in GC

\begin{tabular}{llll}
\hline \multirow{2}{*}{ Factors } & \multicolumn{3}{l}{ Overall survival } \\
\cline { 2 - 4 } & RR & $95 \%$ CI & P \\
\hline Microsatellite status & 3.053 & $1.782-5.231$ & $<0.001$ \\
CD8+M cells density & 1.795 & $1.214-2.655$ & 0.003 \\
CD68+M cells density & 1.082 & $0.677-1.729$ & 0.741 \\
PD1 expression & 1.205 & $0.802-1.809$ & 0.369 \\
PDL1[T] expression & 2.696 & $1.579-4.602$ & $<0.001$ \\
PDL1 expression & 0.867 & $0.560-1.343$ & 0.524 \\
TNM stages & 3.301 & $1.607-6.783$ & 0.001 \\
Infiltration depth & 0.223 & $0.086-0.577$ & 0.002 \\
lymphatic metastasis & 0.372 & $0.185-0.745$ & 0.005 \\
Vessel carcinoma embolus & 0.711 & $0.462-1.094$ & 0.121 \\
\hline
\end{tabular}

RR: relative risk

In our study, we analyzed the correlations between PD1/PDL1 expression and CD8+ $\mathrm{T}$ cell/CD68+ M densities in MSI or MSS GC. We found that PD1 expression was correlated with $\mathrm{CD} 8+\mathrm{T}$ cell density in MSI GC, and PDL1 expression was significantly correlated with high CD68+ M density in MSS GC. It has been reported that the most common tumor-infiltrating immune cells are CD8+ T cells and CD68+ M, accounting for $15 \%$ and $13 \%$ of all tumor cells, respectively. Among the four molecular subtypes of GC classified by TCGA, MSI GC shows high-density infiltration of $\mathrm{T}$ cells and macrophages, among which $\mathrm{T}$ cells account for $30 \%-50 \%$, while macrophages account for $20 \%$, and $70 \%$ of MSI tumors express PDL1 [32]. PDL1 expression is strongly associated with a high density of TILs and MMR deficiency in GC [33]. In a previous study, PDL1 expressed in cancer cells could inhibit the activation of CD8+ CTLs, allowing cancer cells to evade the immune monitor and survive [34]. CXCL8 secreted by macrophages inhibits the function of CD8+ $\mathrm{T}$ cells and participates in the 
immunosuppression tumor microenvironment by inducing PDL1+ macrophages in GC [35]. Furthermore, patients with GC exhibiting higher $\mathrm{T}$ cell infiltration showed increased PD1/PDL1 expression, indicating that an adaptive immune resistance mechanism might exist [36]. In addition, MSI cancer may attract a large number of TILs and exhibit an active immune microenvironment, which leads to the significant upregulation of multiple immune checkpoint proteins, including PD1 and PDL1 [26]. This may explain why MSI tumors respond well to PD1/PDL1 immunotherapy.

The heterogeneity of immune cell distribution within tumors can influence the therapeutic response [37]. The type and quantity of immune cells in the tumor center or invasion front of the tumor can vary greatly; this may explain the ability of these tumors to evade destruction by the human immune system. This phenomenon has been confirmed in colon cancer. Llosa et al. found that large numbers of PDL1+ immune cells, such as macrophages and TILs, were present at the invasion front of MMR defects in colon cancer [38]. Korehisa et al. confirmed that PDL1, CD68+ $\mathrm{M}$, and CD8+ $\mathrm{T}$ cell levels at the invasion frontier region were significantly higher than those in the tumor center of MSI-H colon cancer, and PDL1 was expressed in tumor cells and CD68+/CD163+ (M2) $\mathrm{M}$ at the invasion front of MSI-H colon cancer [39]. However, such studies have not yet been conducted in GC. In our study, CD8+ T cell/CD68+ M densities were evaluated according to the focal location of cancer tissues, including the tumor center and invasion front. The correlations between PD1/PDL1 expression and CD8 $+\mathrm{T}$ cell/CD68 $+\mathrm{M}$ densities in different locations were further analyzed. We found a significant correlation between PD1/PDL1 expression and high CD8+ T cell/CD68+ $M$ density, especially at the invasion front in MSI or MSS GC. Both pathways may contribute to tumor invasion and immune escape in MSI or MSS GC and thus may affect the efficacy of PD1/PDL1 immunotherapy.

This study further analyzed the relationship between PD1/PDL1 expression and CD8+ $\mathrm{T}$ cell/CD68+ M densities and prognosis. Until now, PD1/PDL1 expression in relation to prognosis in GC remains elusive. A meta-analysis showed that PDL1 negativity was associated with better overall survival in GC [24]. Low PD1, PDL1, and CD8 mRNA levels were more significantly associated with a poor prognosis [40]. A study revealed that CD68+ TAMs had no significant effect on OS [41]. In this study, we found that patients with MSI who were PD1 positive, PDL1[T] negative, and PDL1 negative had a better prognosis in GC. Multivariate survival analysis showed that CD8+ $\mathrm{T}$ cell density and PDL1[T] were independent prognostic factors for GC. Our results are consistent with those of a previous study, and the differences involved remain to be further studied.
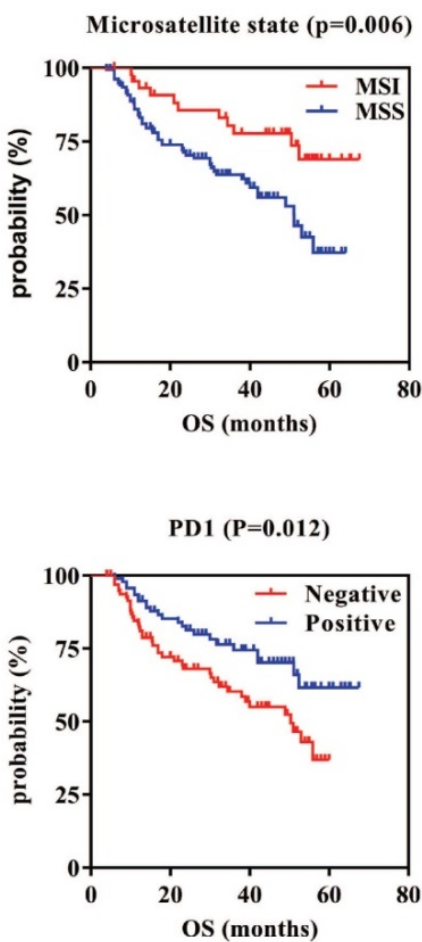

CD8 $+\mathrm{T}$ cell density $(\mathrm{P}=\mathbf{0 . 8 7 0})$

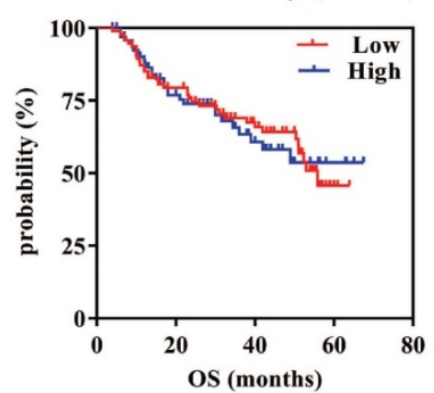

PDL1[T] $(\mathrm{P}=\mathbf{0 . 0 0 5})$

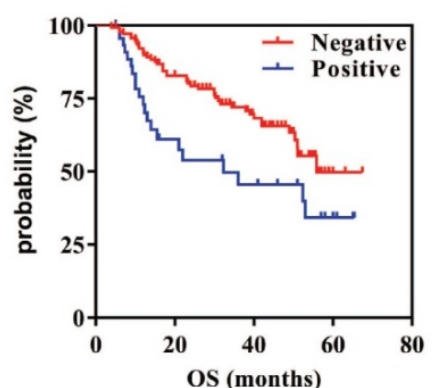

CD68 $+M$ cell density $(P=0.985)$

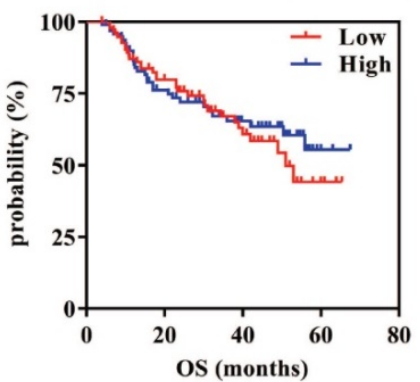

PDL1 ( $\mathrm{P}=\mathbf{0 . 0 2 2})$

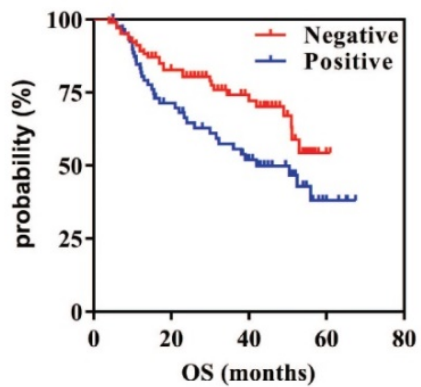

Figure 4 Kaplan-Meier overall survival curves of PDI/PDLI expression or CD8+T cells/CD68+M density in GC. GC patients with MSI, PDI positive, and PDLI[T]/PDL1 negative had better prognosis $(P=0.006,0.012,0.005$ and 0.022 , respectively). There are no correlations between $C D 8+T$ cells $/ C D 68+M$ density and prognosis in $G C(P=0.870$ and 0.985 , respectively). 


\section{Conclusion}

In summary, we evaluated PD1/PDL1 expression and its correlation with CD8+ $\mathrm{T}$ cell/CD68+ $M$ densities in MSI or MSS GC. The results showed that PD1/PDL1 expression and CD8+ $\mathrm{T}$ cell/CD68+ $\mathrm{M}$ densities were higher in MSI GC than in MSS GC, suggesting that PD1/PDL1 expression and the immune response varied based on microsatellite status in GC. Moreover, we found a significant correlation between PD1/PDL1 expression and high CD8+ T cell/CD68+ M density, especially at the invasion front in MSI or MSS GC, suggesting that they may participate in immune escape and affect the efficacy of immunotherapy. Patients with MSI with high PD1/PDL1 expression or high CD8+ $\mathrm{T}$ cell/CD68+ $\mathrm{M}$ densities might be potential beneficiaries of PD1/PDL1 immunotherapy. This study may provide a better understanding of the immune microenvironment in GC with different microsatellite statuses and may provide clues for seeking potential therapeutic targets and prognostic biomarkers for the treatment of GC in clinical settings.

\section{Abbreviations}

GC: gastric cancer; MSI: microsatellite unstable; MSS: microsatellite stable; CD68+ $\mathrm{M}$ : CD68+ macrophage; PD1: programmed cell death protein 1; PDL1: programmed death ligand 1.

\section{Acknowledgements}

\section{Funding}

This study was supported by The National Key R\&D Program of China (2018YFC1311600) and Liaoning Revitalization Talents Program (Grant \#XLYC1808036).

\section{Author's contributions}

Conceived and designed the experiments: Yuan Yuan. Performed the experiments: Yanli Wang and Liang Li. Collected the samples and Analyzed the data: Yanli Wang, Yuehua Gong and Zhi Lv. Contributed reagents/materials/analysis tools: Yuan Yuan. Wrote and revised the paper: Yanli Wang and Yuan Yuan. All authors read and approved the final manuscript.

\section{Competing Interests}

The authors have declared that no competing interest exists.

\section{References}

1. Bray F, Ferlay J, Soerjomataram I, et al. Global cancer statistics 2018: GLOBOCAN estimates of incidence and mortality worldwide for 36 cancers in 185 countries. CA Cancer J Clin. 2018; 68: 394-424.
2. Cancer Genome Atlas Research N. Comprehensive molecular characterization of gastric adenocarcinoma. Nature. 2014; 513: 202-9.

3. Coussens LM, Werb Z. Inflammation and cancer. Nature. 2002; 420: 860-7.

4. Mami-Chouaib F, Blanc C, Corgnac S, et al. Resident memory T cells, critical components in tumor immunology. J Immunother Cancer. 2018; 6: 87.

5. Wang F, Li B, Wei $\mathrm{Y}$, et al. Tumor-derived exosomes induce PD1(+) macrophage population in human gastric cancer that promotes disease progression. Oncogenesis. 2018; 7: 41.

6. Topalian SL, Drake CG, Pardoll DM. Immune checkpoint blockade: a common denominator approach to cancer therapy. Cancer Cell. 2015; 27: 450-61.

7. $\mathrm{Yu}$ Y. Molecular classification and precision therapy of cancer: immune checkpoint inhibitors. Front Med. 2018; 12: 229-35.

8. Marginean EC, Melosky B. Is there a role for programmed death ligand-1 testing and immunotherapy in colorectal cancer with microsatellite instability? Part II-the challenge of programmed death ligand-1 testing and its role in microsatellite instability-high colorectal cancer. Arch Pathol Lab Med. 2018; 142: 26-34

9. Dermani FK, Samadi P, Rahmani G. PD-1/PD-L1 immune checkpoint: Potential target for cancer therapy. J Cell Physiol. 2019; 234: 1313-25.

10. Francisco LM, Salinas VH, Brown KE, et al. PD-L1 regulates the development, maintenance, and function of induced regulatory T cells. J Exp Med. 2009; 206: 3015-29.

11. Baraibar I, Melero I, Ponz-Sarvise M, et al. Safety and tolerability of immune checkpoint inhibitors (PD-1 and PD-L1) in cancer. Drug Safety. 2019; 42: 281-94.

12. Topalian SL, Hodi FS, Brahmer JR, et al. Safety, activity, and immune correlates of anti-PD-1 antibody in cancer. N Engl J Med. 2012; 366: 2443-54.

13. Kim ST, Cristescu R, Bass AJ, et al. Comprehensive molecular characterization of clinical responses to PD-1 inhibition in metastatic gastric cancer. Nat Med. 2018; 24: 1449-58.

14. Passiglia F, Bronte G, Bazan V, et al. PD-L1 expression as predictive biomarker in patients with NSCLC: a pooled analysis. Oncotarget. 2016; 7: 19738-47.

15. Patel SP, Kurzrock R. PD-L1 expression as a predictive biomarker in cancer immunotherapy. Mol Cancer Ther. 2015; 14: 847-56.

16. Le DT, Durham JN, Smith KN, et al. Mismatch repair deficiency predicts response of solid tumors to PD-1 blockade. Science (New York, NY). 2017; 357: 409-13.

17. Zhao $\mathrm{P}, \mathrm{Li} \mathrm{L}$, Jiang $\mathrm{X}$, et al. Mismatch repair deficiency/microsatellite instability-high as a predictor for anti-PD-1/PD-L1 immunotherapy efficacy. J Hematol Oncol. 2019; 12: 54.

18. Feng X, Liu J, Gong $\mathrm{Y}$, et al. DNA repair protein XPA is differentially expressed in colorectal cancer and predicts better prognosis. Cancer Med. 2018; 7: 2339-49

19. Takada K, Okamoto T, Shoji F, et al. Clinical significance of PD-L1 protein expression in surgically resected primary lung adenocarcinoma. J Thorac Oncol. 2016; 11: 1879-90

20. Baretti M, Le DT. DNA mismatch repair in cancer. Pharmacol Ther. 2018; 189: $45-62$

21. Jin Z, Yoon HH. The promise of PD-1 inhibitors in gastro-esophageal cancers: microsatellite instability vs. PD-L1. J Gastrointest Oncol. 2016; 7: 771-88.

22. Ilson DH. Advances in the treatment of gastric cancer. Curr Opin Gastroenterol. 2018; 34: 465-8.

23. Fashoyin-Aje L, Donoghue $\mathrm{M}$, Chen $\mathrm{H}$, et al. FDA approval summary: pembrolizumab for recurrent locally advanced or metastatic gastric or gastroesophageal junction adenocarcinoma expressing PD-L1. Oncologist. 2019; 24: 103-9.

24. Gu L, Chen M, Guo D, et al. PD-L1 and gastric cancer prognosis: A systematic review and meta-analysis. PLoS One. 2017; 12: e0182692.

25. Bailey MH, Tokheim C, Porta-Pardo E, et al. Comprehensive characterization of cancer driver genes and mutations. Cell. 2018; 173: 371-85.e18.

26. Nebot-Bral L, Coutzac C, Kannouche PL, et al. Why is immunotherapy effective (or not) in patients with MSI/MMRD tumors? Bull Cancer. 2019; 106: 105-13.

27. Bernal M, Concha A, Saenz-Lopez P, Rodriguez AI, Cabrera T, Garrido F, et al. Leukocyte infiltrate in gastrointestinal adenocarcinomas is strongly associated with tumor microsatellite instability but not with tumor immunogenicity. Cancer Immunol Immunother. 2011; 60: 869-82

28. Shin SJ, Kim SY, Choi YY, et al. Mismatch repair status of gastric cancer and its association with the local and systemic immune response. Oncologist. 2019; 24: e835-e44.

29. Angell HK, Lee J, Kim KM, et al. PD-L1 and immune infiltrates are differentially expressed in distinct subgroups of gastric cancer. Oncoimmunology. 2019; 8: e1544442.

30. De Smedt L, Lemahieu J, Palmans S, et al. Microsatellite instable vs stable colon carcinomas: analysis of tumour heterogeneity, inflammation and angiogenesis. Br J Cancer. 2015; 113: 500-9.

31. Kim Y, Wen X, Bae JM, et al. The distribution of intratumoral macrophages correlates with molecular phenotypes and impacts prognosis in colorectal carcinoma. Histopathology. 2018; 73: 663-71.

32. Kim TS, da Silva E, Coit DG, et al. Intratumoral immune response to gastric cancer varies by molecular and histologic subtype. Am J Surg Pathol. 2019; 43: 851-60.

33. Kawazoe A, Kuwata T, Kuboki Y, et al. Clinicopathological features of programmed death ligand 1 expression with tumor-infiltrating lymphocyte, 
mismatch repair, and Epstein-Barr virus status in a large cohort of gastric cancer patients. Gastric Cancer. 2017; 20: 407-15.

34. Kumar R, Yu F, Zhen $\mathrm{YH}$, et al. PD-1 blockade restores impaired function of ex vivo expanded CD8(+) T cells and enhances apoptosis in mismatch repair deficient EpCAM(+)PD-L1(+) cancer cells. Onco Targets Ther. 2017; 10: 3453-65.

35. Lin $\mathrm{C}, \mathrm{He} \mathrm{H}$, Liu $\mathrm{H}$, et al. Tumour-associated macrophages-derived CXCL8 determines immune evasion through autonomous PD-L1 expression in gastric cancer. Gut. 2019; 68: 1764-73.

36. Xing X, Guo J, Ding G, et al. Analysis of PD1, PDL1, PDL2 expression and T cells infiltration in 1014 gastric cancer patients. Oncoimmunology. 2018; 7: e1356144.

37. Junttila MR, de Sauvage FJ. Influence of tumour micro-environment heterogeneity on therapeutic response. Nature. 2013; 501: 346-54.

38. Llosa NJ, Cruise M, Tam A, et al. The vigorous immune microenvironment of microsatellite instable colon cancer is balanced by multiple counter-inhibitory checkpoints. Cancer Discov. 2015; 5: 43-51.

39. Korehisa S, Oki E, Iimori M, et al. Clinical significance of programmed cell death-ligand 1 expression and the immune microenvironment at the invasive front of colorectal cancers with high microsatellite instability. Int J Cancer. 2018; 142: 822-32.

40. Ito S, Masuda T, Noda M, et al. Prognostic significance of PD-1, PD-L1 and CD8 gene expression levels in gastric cancer. Oncology. 2020; p: 1-11.

41. Yin S, Huang J, Li Z, et al. The prognostic and clinicopathological significance of tumor-associated macrophages in patients with gastric cancer: a meta-analysis. PLoS One. 2017; 12: e0170042. 